\title{
Are Solid Catalysts Successfully Emulating Enzymes?
}

\author{
Bernard DELMON* \\ Université catholique de Louvain, CATA, Catalysis and Chemistry of Disperse Materials, Place Croix du Sud 2/17, \\ B-1348 Louvain-la-Neuve, Belgium
}

\begin{abstract}
In many aspects, similitude exists between man-made catalysts and enzymes. Can scientific insight into this similitude stimulate new research directions in catalysis? More precisely, can the understanding of the mechanisms of activity control in enzymes suggest new advances for man-made catalysts? Indeed, it is already possible to design catalysts with (i) new structures (e.g. presence of several phases) and (ii) a better balance between the roles of the different components. The ambition is to keep the essential advantages of solids as catalysts, in particular robustness, easy separation from products, and tolerance to high temperatures, while adding the advantages typical of enzymes, like more activity, allostery, and more precise control of selectivity, in particular enantioselectivity. The ambition is to build a bridge between robust man-made functional solids and fragile but extremely selective enzymes. For that, results from a line of the work in our group will be used.
\end{abstract}

Key words: inorganic catalyst; enzyme; activity; selectivity

CLC number: O643 Document code: A

Industry is repeatedly challenging investigators to build bridges between heterogeneous catalysis (HET) on the one hand, and homogeneous (HOM) or enzymatic (ENZ) catalysis on the other hand. Scientists responsible for industrial development do not seem satisfied by the response of the scientific community. This may be because during congresses or in the scientific literature, scientists and engineers working in heterogeneous catalysis failed to highlight the many similarities between the two different fields. This is prejudicial to a more creative development of catalysis.

Actually, discoveries are being progressively made that the catalysts developed by modern technology possess many of the characteristic properties of enzymes. In particular, a control of catalytic activity is often exerted by special parts of the catalyst structure situated at some distance from the catalytic site. This action is similar to that of the regulating part of the large family of regulating enzymes on a prosthetic (active) site. Another discovery concerning inorganic catalysts was their ability to change form, which is a phenomenon common to all enzymes. Both phenomena have been observed in groups of inorganic catalysts of considerable importance in petroleum refining (hydrotreatment), petrochemistry (selective oxidation and ammoxidation), and the elimination of dangerous effluents (complete oxidation).

The present contribution is an attempt by a member of the HET community to highlight the important bridges that already exist. It also offers speculations concerning future scientific or technical developments. To achieve this goal, features common to heterogeneous and other categories of catalysis, i.e., enzymatic, and occasionally homogeneous catalysis, will be examined. The discussion will start by focusing on processes that operate in the HET 'group' that also operate in the other, ENZ or HOM, groups. However, some fields will not be considered because suitable examples accessible to scientific scrutiny are too few or involve very complex arguments. This includes the fields of oxidative dehydrogenation, ammoxidation, complete oxidation for the elimination of harmful materials, catalysis by soluble catalysts, etc., although similar interpretations of their action are often possible.

A first point is that our culture of physical chemistry has perhaps been too inflexible about the rigidity of solids. Our concept of active sites uses the picture that surfaces were frozen. Actually, catalytic reactions are dynamic processes and too little attention has been given to the many consequences of this. A typical example that we shall comment on concerns results where the catalytic reaction creates holes (pits or 'nests') in the solid. The reacting molecules penetrate into these pits where they undergo changes while they simultaneously modify the bonds in the 'solid' catalyst thereby altering the composition of the nest. The nests that have been characterized in this way have dimensions ranging from nanometers to several micrometers.

Enzymes are the result of evolution in nature. They may therefore be considered as perhaps "perfect" catalysts created by living species in their struggle for life during evolu-

Received date: 23 April 2010.

*Corresponding author. Tel: +32-10-473590; Fax: +32-10-473649; E-mail: Bernard.Delmon@uclouvain.be

English edition available online at ScienceDirect (http://www.sciencedirect.com/science/journal/18722067). 
tion. A line of thought is to consider the development of catalytic concepts starting from the Philosopher's Stone of the alchemists in Europe in the Middle Ages to modern catalysts and man-made catalysts that possess enzyme-like properties. The feature common to modern catalysts and the Philosopher's Stone is the complexity of the preparation and, frequently, the secrecy surrounding it. The situation becomes much more stimulating when man-made catalysts in laboratories and industry are compared to working enzymes or synthetic molecules with catalytic activity.

\section{The complexity of solid catalysts}

The fact that catalysts are complex was recognized very soon after the discoveries of Paul Sabatier (Nobel Prize 1912). His catalysts were simple metals that catalyzed the hydrogenation of relatively simple molecules. Roughly speaking, Sabatier's views were that the catalyst formed an intermediate compound that liberated the products when it decomposed. This is the catalytic cycle ('a catalyst is a solid that accelerates reactions by a cycling process without being altered at the end of the cycle' which is the best definition of catalysis). The other content in terms of explanation was that the catalytic activity was due to the solid surface.

The works of Karl Bosch and Fritz Haber, which were recognized by their Nobel Prizes in 1919, led to a major jump in the concepts concerning catalysis. This was the development of industrial ammonia synthesis. The discovery of the catalytic nature of the reaction was the crucial step, but the development of a viable process needed a further discovery. This was that the incorporation of minor components in the catalysts was essential for making the catalysts more highly active not only in the laboratory but also in large scale production processes. This constitutes one of the many examples where practical applications have driven the progress of catalytic science. Such doping of catalysts has probably been the main line for progress in catalysis in the last hundred years, but explanations turned out to be difficult and were the origin of many controversies. The reason is that they depended on the development of science in other fields. The electronic properties of solids, and the development of transistors in particular, provided an important input for scientific understanding.

In other active and selective catalysts, the existence of more than one single solid phase was essential. This necessitated other new interpretations. Some of them were straightforward, e.g., reaction of hydrogenation by the metals plus role of the acidity of the support in bifunctional catalysts and the case of catalysts that activate consecutive reactions. However, the particular case of the synergy between two phases in a simple one-step hydrogenation, presented by Georg-Maria Schwab in 1970, raised new prob- lems. Another case was the existence of two or several phases in the ammoxidation catalysts of SOHIO (Standard Oil of Ohio). Although this was detected very early by the scientists of that company, it was not properly noticed and the company did not mention data indicating that it was important for the catalyst to contain two or more phases until the year 2000. The doping of the catalytic phase by minute amounts of foreign ions remains the major basis for interpretation in the scientific literature. Other ideas started from the picture of an electronic junction between the particles (a sort of transistor). This zone of contact was supposed to be the origin of catalytic activity. But this view is obviously too simple because the fraction of the solids accessible to reactants would be very small since it would consist only of the one-dimensional intersection of the junction formed between the surface extending into the interior with the outside solid surface. It is only recent progress in making nano-particles that has permitted the investigation of the structure of the junction on the atomic scale and allowed the identification of such effects.

\section{Cooperation between different phases in ca- talysis}

In enzymology, a special class of enzymes possesses the property of allostery. They are called regulating enzymes (Fig. 1). Their activity changes as a function of the composition of the surrounding medium. An interesting observation concerned the place where the surrounding medium acted to perform the regulation, e.g. by the adsorption of a molecule. This was situated at a substantial distance from the catalytic ('prosthetic') site. Some sort of 'message' must necessarily be sent from the adsorption site to the prosthetic place. A similar phenomenon was successively discovered in several types of solid catalysts. This occurred shortly after the observations of Georg-Maria Schwab.

Returning to processes occurring in solid catalysts, zeolite catalysts raise interesting problems. They have an important role in a group of reactions that involve breaking some bonds and/or transforming the structure of hydrocarbon molecules in the presence of hydrogen (hydrocracking and/or hydroisomerization). This role needs an interpretation that also explains that these catalysts contain two separate parts: the zeolite lattice, on the one hand, and a metal, on the other hand, that was deposited on the zeolite surface. The metal frequently used was platinum. This metal is able to activate hydrogen and catalyze both hydrogenation and dehydrogenation reactions. However, hydrocracking and hydroisomerization also need acid or acid-base sites. The superiority of zeolites was due to that their acidity allowed both reactions to occur and permitted the selective formation of products. The latter was attributed to the role of pore 


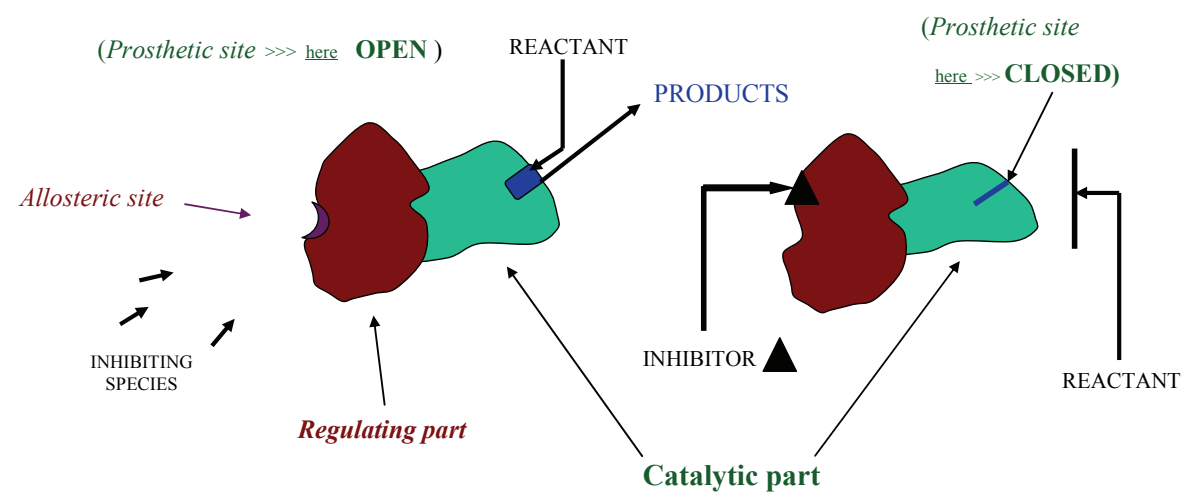

Fig. 1. Schematic representation of a regulating enzyme in its active and inhibited states. The phenomenon of change is called allostery.

diffusion. The structure of the zeolites enabled 'sieving' of the useful species. The metal was mainly deposited on the surface of the zeolite crystallites, but the reaction took place inside the cages. This led to the hypothesis that acid sites were situated inside in the pore lattice. In mechanistic studies, the hypothesis used was that hydrogen in the form of protons was involved. It was known that the noble metal can break the $\mathrm{H}-\mathrm{H}$ bond. The picture thus was that a hydrogen species migrated to the interior of the zeolite and formed Brönsted $-\mathrm{OH}$ sites (or modified their acid strength). Quite naturally, this mechanism was not readily accepted and many other explanations were proposed.

A serious objection was that the effect was not due to a modification of the catalytic sites and hydrogen was involved directly in two or several of the reactions of the reactant (dehydrogenation-hydrogenation). These reactions also occur in hydrocracking and hydrotreatment. However, the mechanism used in the objection cannot explain the isomerization of compounds containing only saturated $\mathrm{C}-\mathrm{C}$ bonds and aromatic rings. In particular, with mordenite [1] or pillared clays [2], an extensive and rapid transalkylation of $o$-xylene to the $m$ - and $p$ - isomers was observed, and this occurred only if spillover hydrogen produced by a metal was present.
At about the same time, the formation of special forms of hydrogen that were very mobile on surfaces was discovered. This is the spillover phenomenon. Migration has been demonstrated in experiments performed to prove the existence of spillover. Important evidence for the existence of this phenomenon will be presented in subsequent paragraphs. A group of measurements are of particular interest. Direct investigations on the mobile species were made by a group of investigators including U. Roland, K-H. Steinberg, F. Roessner, and H. G. Karge. They assembled data supporting the existence of a spillover mechanism [3-6]. But the existence of an electric charge on the mobile species was still debated. The 'spiltover' hydrogen will be called $\mathrm{H}_{\mathrm{so}}$. The merit of these authors was to show that (i) this spillover took place inside zeolites and (ii) the migrating hydrogen species were electrically charged. The experimental setup comprised a succession of tablets in contact with each other (Fig. 2). During the reaction, hydrogen reacts with $\mathrm{Pt}$ on the top, while the catalytic reaction occurs on the zeolite side situated at the bottom. When a charged particle moves in a magnetic field, a force pushes it in a direction perpendicular to the movement. The experiment detected a polarization of the pure zeolite pellet in this direction when a reaction took place at the bottom. This con-

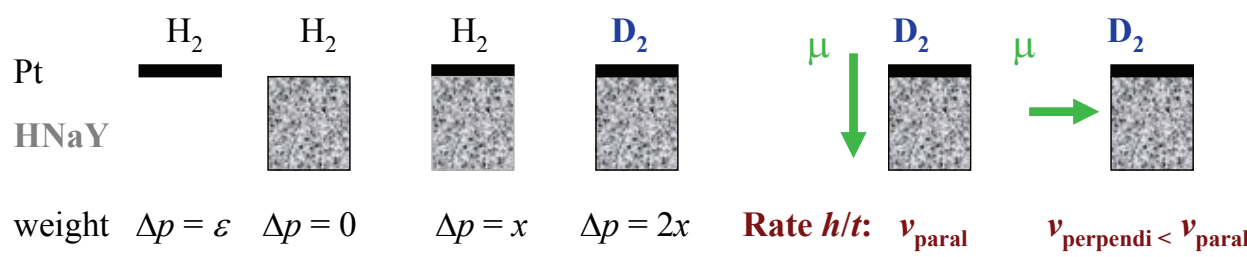

Fig. 2. Schematic representation of the migration of spillover hydrogen or deuterium produced on a platinum-containing layer (left), from this layer to the bottom part of a $\mathrm{HNaY}$ zeolite where an acid catalyzed reaction took place. (i) $\Delta p$ : differences in weight when hydrogen or deuterium was introduced and (ii) modification of the vertical migration rate $v=h / t$ of the charged species as detected by the potential difference between the sides of the sample when the latter was subjected to a magnetic field $\mu$ parallel or perpendicular to the direction of migration. The rate was lower when the magnetic field was perpendicular to the direction of migration. 
firmed the formation of charged species. An extremely sensitive weighing device was used that could detect the migration by taking advantage of the heavier atomic weight of deuterium. This is presented schematically in Fig. 2.

All these effects have a strong analogy with allostery in enzymes. A signal emitted by one of the phases creates or modifies the active sites situated in another part. On the basis of different experiments, other properties of spillover hydrogen led to the conclusion that the species was a proton. It is therefore logical to say that the signal triggered processes involve the movement of electronic densities. This is also the situation in the present case of Brönsted sites inside zeolites, which were created by the action of a charged mobile species.

\section{3 'Communication' between different solid phases and creation of special sites}

Historically, the reactions of solids gave the first models of reactions in which spillover species of hydrogen produced observable chemical effects. A nickel oxide powder can be reduced by hydrogen to metallic Ni at normal pressure and temperatures as low as $180{ }^{\circ} \mathrm{C}$ when mixed with particles of platinum or other metals ( $\mathrm{Pd}, \mathrm{Cu}$, etc.). In this way, at $200{ }^{\circ} \mathrm{C}$, a sample can be completely reduced in 30 min, whereas no reduction can be detected in the absence of the foreign metals $[7,8]$. The mechanism of the reaction is schematically represented in Fig. 3. Figure 3(a) suggests the mechanism by which spillover hydrogen can move on the surface of nickel oxide by a continuous exchange of electrons between the proton and $\mathrm{Ni}^{3+}$ defects. $\mathrm{NiO}$ prepared at moderate temperature is a semiconductor because its lattice

(a)

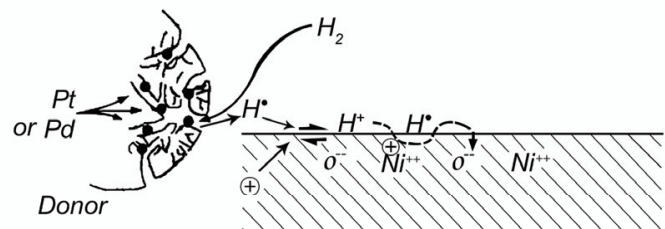

$\mathrm{Ni}^{++} \oplus \mathrm{o}^{-}, \mathrm{O}^{-}$

(b)

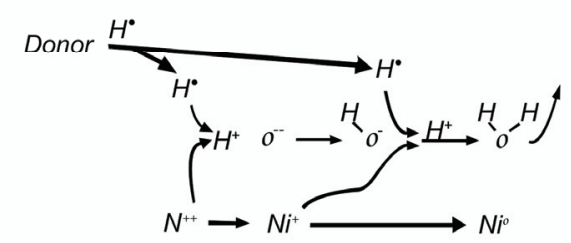

Fig. 3. (a) Suggested mechanism of the movement of spillover hydrogen $\mathrm{H}_{\text {so }}$ by the hopping of charged species between the surface and electronic defects in a semiconducting solid. There are evidence that the surface hydrogen is that of the $-\mathrm{OH}$ of Brönsted acid sites. (b) Mechanism of the reduction of $\mathrm{NiO}$ by the two-step formation of $\mathrm{H}_{2} \mathrm{O}$. contains a few $\mathrm{Ni}^{+++}$ions together with the normal $\mathrm{Ni}^{++}$ions (its true formula is $\mathrm{NiO}_{1+x}$ ). The reduction of $\mathrm{Ni}^{++} \mathrm{O}^{--}$to the metal is due to the two-step reduction of $\mathrm{Ni}^{++}$to $\mathrm{Ni}^{0}$ and the formation of $\mathrm{H}_{2} \mathrm{O}$ (Fig. 3(b)). A similar reduction of metal oxides in the presence of $\mathrm{H}_{\mathrm{so}}$ was also observed in the complete transformation of, respectively, $\mathrm{CuO}$ and $\mathrm{Co}_{3} \mathrm{O}_{4}$ to $\mathrm{Cu}$ and $\mathrm{Co}$, and other oxides to sub-oxides (e.g., $\mathrm{MoO}_{3}$ or $\left.\mathrm{V}_{2} \mathrm{O}_{5}\right)[7,8]$.

Many direct proofs of the migration of hydrogen on surfaces have been obtained. As in the measurements presented previously, use was made of deuterium because its chemical properties are identical to those of hydrogen, while its other properties permit a distinction, e.g., in infrared spectra where $\mathrm{OH}$ and $\mathrm{OD}$ vibrations appear at different wavenumbers. In several experiments, the sample was a silica support, the surface of which was covered by - $\mathrm{OH}$ groups, and small particles of platinum were placed on the support. Using a narrow slit on the spectrometer, it was possible to observe the apparition and migration of a deuterium band from the silica initially covered by $-\mathrm{OH}$. The rate of progress with time of the boundary between areas covered by -OD and $-\mathrm{OH}$ was measured [9]. This observation and other similar data confirmed the validity of the mechanism concerning the control of Brönsted acidity in zeolites.

Several review papers have described results obtained in reactions of solids and in catalysis, e.g., references [10,11]. These articles showed that mobile, or spillover, species can exert a control on the catalytic activity and selectivity of catalysts in a way that is conceptually similar to the control processes exhibited by enzymes. The illustration of this is possible in several types of reactions. Some of them are indicated below.

\section{The control of catalytic activity and selectivity thanks to spillover signals: the 'remote control'}

The results already presented are of interest for the development of concepts, but are present for a limited number of reactions of solids. The situation is different in catalysis where spillover hydrogen plays an important role in the continuous elimination of hydrocarbon deposits during catalytic reactions. This is well documented in the literature. The design of new catalysts takes into consideration this phenomenon and has been done with success.

However, still more important for understanding the activity and mechanism of reactions is the creation of new catalytic sites. These sites are subjected to the control of 'messengers', or signals, produced by specific parts of the complex catalysts. These signals can be modulated by the regulation of their production by the controlling phase. We shall call these controlling phases 'donors', and the regulated parts of the catalysts, 'acceptors' of the signal. In hy- 
droisomerization/hydrocracking, the donor of $\mathrm{H}_{\mathrm{so}}$ is platinum, and the acceptor, a zeolite. In allosteric enzymes (also called regulating enzymes), a part of the enzymatic structure adsorbs the regulating substance (product of the reaction, acid or basic molecules, etc.) and sends a signal. The switching on or off of the active sites (named 'prosthetic') upon receiving a signal is the response. Although there are differences in the mechanisms, regulation must therefore be considered a feature common to solid catalysts and enzymes

In this section, signals consisting of spillover species will be considered. These may be spillover hydrogen, $\mathrm{H}_{\mathrm{so}}$, or mobile oxygen species, $\mathrm{O}_{\mathrm{so}}$. One example of limited practical interest concerning oxygen will be mentioned first because the reaction is simple and the phenomenon only permits one single interpretation. The other examples concern two groups of extremely important reactions in industry: a family of selective oxidations and the hydrotreatment of fuels. It can be mentioned that the movement of other chemical particles has also been tentatively considered in the literature.

\subsection{A simple reaction influenced by a remote control process: an oxygen-assisted dehydration}

In this example, the catalyst is composed of a simple mixture of molybdenum and antimony oxides, symbolized by $\left\{\mathrm{MoO}_{3} / \mathrm{Sb}_{2} \mathrm{O}_{4}\right\}$. The acidity of the mixture is due, in strength and amount, to that of the $\mathrm{MoO}_{3}$ content, either pure or in the mixture when there are no molecules that can be oxidized. If the mixture is contacted with air at the temperature at which the reaction studied takes place, this acidity increases considerably [11-13]. A spillover species of oxygen $\mathrm{O}_{\mathrm{so}}$ creates additional acid sites (actually Brönsted sites). The movement of $\mathrm{O}_{\text {so }}$ and the 'active sites' created (-OH sites in the present case) are represented schematically in Fig. 4. There is no contamination of one oxide by metal elements of the other oxide [12,13].

The very simple reaction catalyzed by the $\left\{\mathrm{MoO}_{3} / \mathrm{Sb}_{2} \mathrm{O}_{4}\right\}$ mixture is the dehydration of $N$-ethyl-formamide with rearrangement to acetonitrile:

$$
\mathrm{C}_{2} \mathrm{H}_{5} \text {-NH-CHO } \stackrel{\left\{\mathrm{MoO}_{3} / \mathrm{Sb}_{2} \mathrm{O}_{4}\right\}, \mathrm{O}_{\mathrm{so}}}{\longrightarrow} \mathrm{C}_{2} \mathrm{H}_{5}-\mathrm{CN}+\mathrm{H}_{2} \mathrm{O}
$$

Antimony oxide is not active for this reaction. With the two-phase mixture in the absence of oxygen, the yield is low. A surprising fact is that the yield is considerably increased when the reaction is carried out in an atmosphere containing oxygen. No oxygenated product is detected in the reaction. The remarkable feature of this reaction is that oxygen is neither consumed nor produced in measurable amounts. The effects observed were accounted for by a model with a mechanism of remote control. The agreement between theoretical values (solid line) and experimental

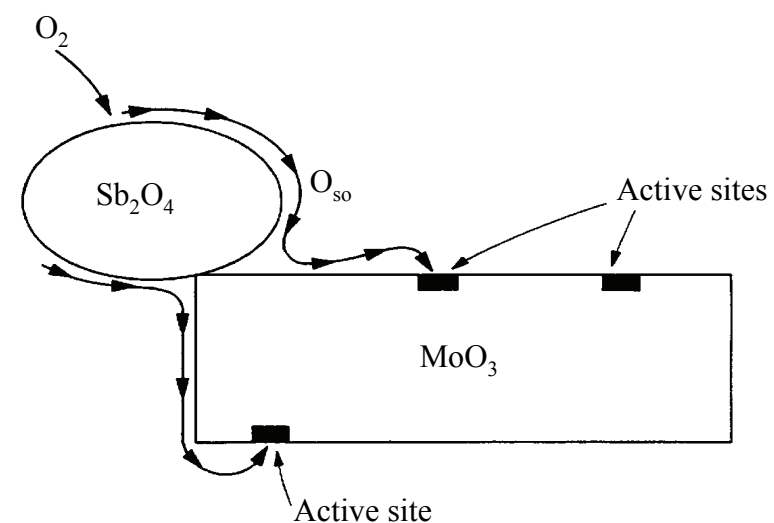

Fig. 4. Schematic representation of the creation of catalytic sites by spillover oxygen $\mathrm{O}_{\text {so }}$ on $\mathrm{MoO}_{3}$. The catalytic sites are $-\mathrm{OH}$ groups of Brönsted acid sites in the case of oxygen assisted dehydration of $\mathrm{N}$-ethylformamide with rearrangement to acetonitrile (and possibly also partially reduced parts of the surface of $\mathrm{MoO}_{3}$ ), as explained in Section 5.1 (Figs. 9, 10, and 11).

points is excellent for different catalyst compositions and different oxygen partial pressures [13]. An example is presented in Fig. 5. The investigations did not permit the physical detection of a difference between 'native' and spillover-created active sites. It is interesting to note that this result concerning $\mathrm{O}_{\text {so }}$ is similar to those of spillover hydrogen $\mathrm{H}_{\mathrm{so}}$ in the creation of acid sites, particularly in the isomerization of $o$-xylene mentioned previously, which is one where there was no other reaction (dehydrogenation or hydrogenation).

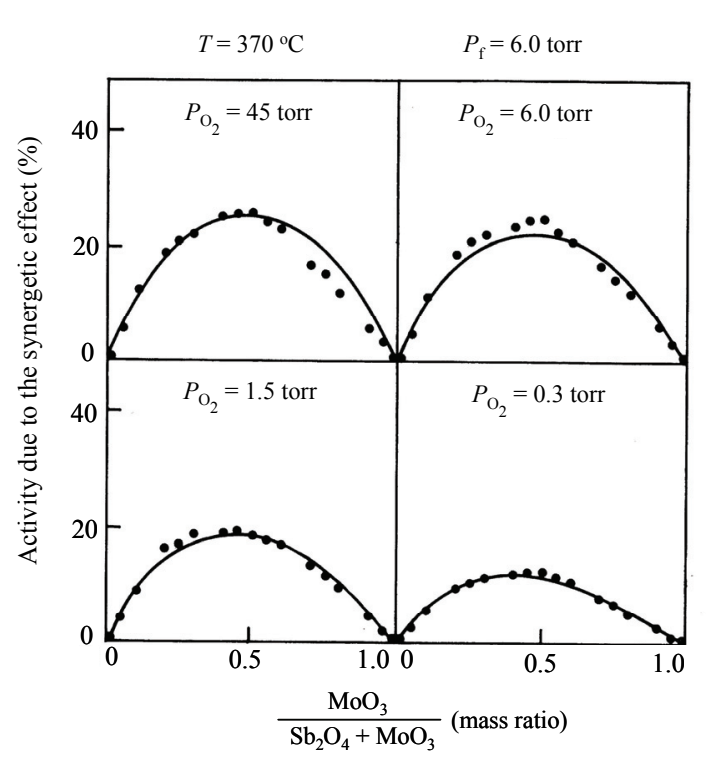

Fig. 5. Oxygen assisted dehydration of $N$-ethylformamide, under the influence of $\mathrm{O}_{\text {so }}$ showing the activity vs. composition of the $\mathrm{MoO}_{3} / \mathrm{Sb}_{2} \mathrm{O}_{4}$ mixture. The theoretical curves based on the remote control mechanism [13] are presented together with the experimental points. 
This kind of effect can explain the results of Georg-Maria Schwab described in the introductory remarks, but other cooperation effects have also been discovered. This shows that the similitude between solid catalysts and enzymes deserves more than a casual remark.

Many selective oxidation or oxidative dehydrogenation reactions of hydrocarbons by molecular oxygen occur also on mixtures of oxides. This is the case with ammoxidation reactions, where mixtures of oxygen and ammonia react with hydrocarbons. Examples are presented in several publications $[10,11,14,15]$. For a long period, the remote control mechanism was not accepted by the majority of investigators. It has now been recognized, since the beginning of the 21 st century, and takes the less precise name of phase co-operation. The very complicated catalysts used for the selective ammoxidation of propane comprise two or several phases. Their inter-mixing has been proved by analyses (X-ray diffraction, infrared spectroscopy, and other techniques) both of the catalysts after they had transformed reactants under normal reaction conditions and by observations during the catalytic phenomenon in special instruments (the so-called in situ or in operando techniques). In the case of the ammoxidation of propane to acrylonitrile, $\mathrm{Sb}_{2} \mathrm{O}_{4}$ is frequently detected, together with $\mathrm{X}_{x} \mathrm{Y}_{y} \mathrm{O}_{z}$ oxides (the elements $\mathrm{X}$ and $\mathrm{Y}$ being $\mathrm{Bi}, \mathrm{Ni}, \mathrm{Cu}, \mathrm{Fe}, \mathrm{Mg}, \mathrm{P}, \mathrm{Sb}, \mathrm{Sn}$, $\mathrm{Te}, \mathrm{W}$, or $\mathrm{Zn})$.

\subsection{Hydrogen-assisted hydroconversion of petroleum fractions: hydrodesulfurization and hydrotreatment}

The hydrotreatment of petroleum fractions is the most important process in refining. This is actually a group of processes used to eliminate the harmful hetero-atoms of sulfur, nitrogen, lead, arsenic, vanadium, etc. (hydropurification) and to reduce the size and modify the structure of molecules contained in the products of distillation (hydrocracking and hydroisomerization). A typical catalyst of this family contains sulfides of cobalt or nickel (or other elements) and sulfided molybdenum or tungsten. The synergy between these two components is the key to the development of refining processes. This was especially the case with the association between cobalt and molybdenum sulfides ('CoMo') deposited on different carriers. We have shown that the sulfides of cobalt, nickel or more or less sulfided elements such as platinum, produce spillover hydrogen. $\mathrm{H}_{\mathrm{so}}$ creates catalytic sites on the sulfide crystallites of Group VI elements [10,11,15-17]. The sketch in Fig. 6 represents the process. Two different sites are created.

These sites differ by the number of sulfur atoms surrounding them, which is suggested in the figure (the presence of the hydrogen atom featured in the bottom part has not been proved but it is likely since the whole surface is

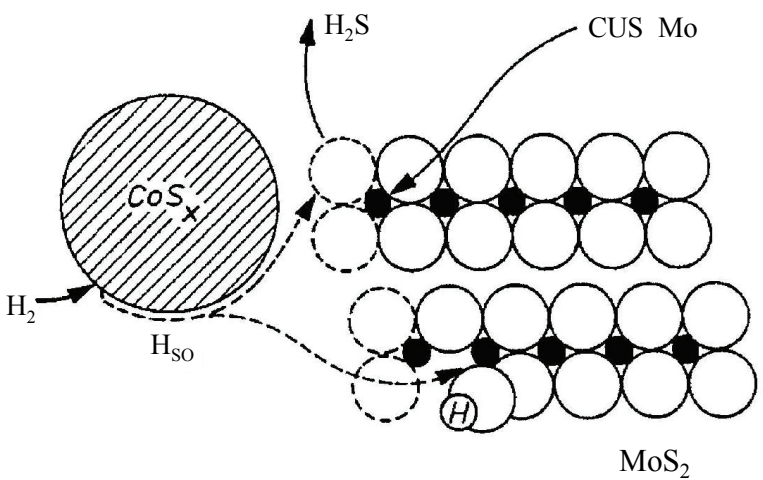

Fig. 6. Schematic representation of the creation of catalytic sites on the edges of $\mathrm{MoS}_{2}$ crystallites by removal of sulfur atoms and/or fixation of $\mathrm{H}_{\mathrm{so}}$. Mild reduction creates molybdenum sites derived of from a sulfur atom normally attached to the structure (coordinatively unsaturated sites, CUS). More deeply reduced sites are either such sites with an attached $\mathrm{H}$ or sites corresponding to a deeper unsaturation.

continuously fed with the hydrogen needed both by the reaction and the production of $\mathrm{H}_{\mathrm{so}}$ ). Two kinds of reactions of hydrogen take place on the surface of remote-controlled $\mathrm{MoS}_{2}$. These are, respectively, the hydrogenation of unsaturated functions (double bonds and aromatic structures), and the hydrogenolysis of bonds between hetero-atoms (especially sulfur) and the hydrocarbon chain (hydropurification, HPUR).

The action of remote control is similar to allostery in enzymes in several aspects. Catalytic sites are created, as in the dehydration example, and a control of selectivity is exerted, but now by the action of $\mathrm{H}_{\mathrm{so}}$ as mobile species. This is shown in Fig. 6. Spillover hydrogen removes sulfur atoms from the lateral planes of $\mathrm{MoS}_{2}$ and creates coordinatively unsaturated sites 'CUS' located on a mildly reduced sulfide surface $[10,11,15-17]$. A very limited degree of unsaturation corresponds to the creation of hydrogenation sites HYD, and the more extensive removal of $\mathrm{S}$ atoms permits purification (HPUR), i.e., the removal of harmful elements. This is important for practical applications because although purification is essential and useful, the saturation of $\mathrm{C}=\mathrm{C}$ double bonds and aromatic rings will diminish the quality of the fuels. The relative number of deeply reduced CUS depends on the amount of $\mathrm{H}_{\mathrm{so}}$ irrigating $\mathrm{MoS}_{2}$. This explains why a higher proportion of donors (Group VIII metal sulfides) and a higher hydrogen pressure advantageously favor hydropurification at the expense of hydrogenation (see Refs. [10] and [11], and references therein). Two parameters control the activity and selectivity: catalyst composition and hydrogen partial pressure (more precisely the $\mathrm{H}_{2} / \mathrm{H}_{2} \mathrm{~S}$ ratio). The following effects were observed:

Group VI/Group VIII metals proportion increase $\Rightarrow$ HPUR/HYD increases

Hydrogen pressure increases $\Rightarrow$ HPUR/HYD increases 
This is shown in Fig. 7. Experimental curves were obtained as a function of the proportion of active elements. The conversions are plotted as a function of the donor/acceptor ratio. The two different curves represent, respectively, the conversions in each group of reactions. They have shapes similar to those presented in Fig. 5 for the oxygen-assisted dehydration of formamide. In conformity with prediction, the maximum of the curve corresponding to HYD appeared at lower proportions of the Group VIII/ Group VI elements as compared to the case of HPUR. Similar results have been reproduced very often in the literature [11, and references therein].

The occurrence of the spillover phenomenon in hydrotreating was not easily accepted by the scientific community. The observed synergy between Group VI and Group VIII was attributed to the formation of a new compound ('CoMoS') $[18,19]$. This probably reflected the hold of traditional concepts although there is no proof of the existence

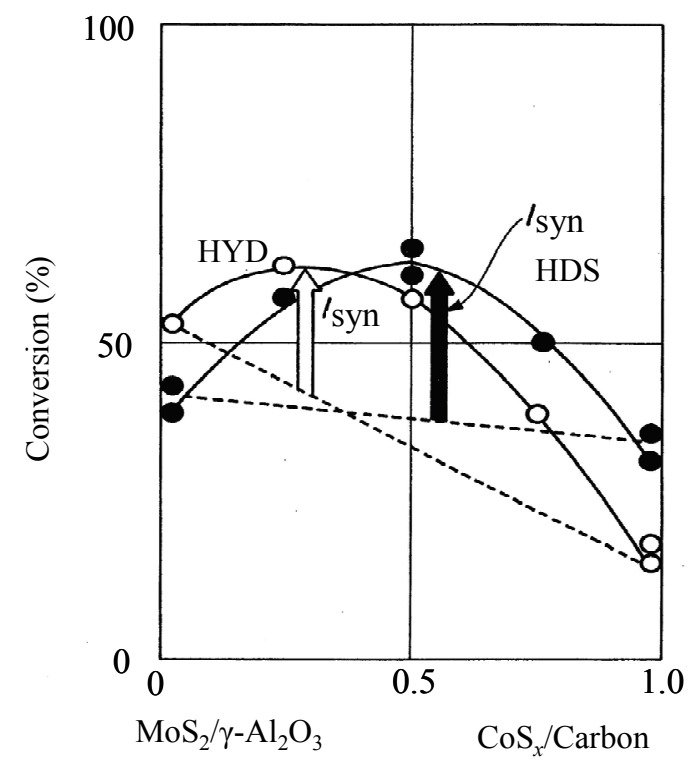

Fig. 7. Typical curves corresponding to the reactions with hydrogen of a mixture of cyclohexene (hydrogenation, HYD) and thiophene (hydrogenolysis, i.e. hydrodesulfurization, HDS) on powder mixtures of, respectively, Mo and Co catalysts. $\mathrm{MoS}_{2}$ was supported on alumina, as Mo in hydrodesulfurization catalysts normally is. Cobalt sulfide, probably slightly reduced $\mathrm{C}_{9} \mathrm{~S}_{8}$, is supported on carbon (Co may be partially transformed to cobalt aluminate when supported alone on a normal alumina support). The reaction was conducted under typical hydrotreatment conditions $\left(300^{\circ} \mathrm{C}, 3 \mathrm{MPa}\right.$ total pressure). The result constitutes a typical example of the displacement of the maximum of the HDS curve for catalysts containing more cobalt, with respect to the HYD curve. The same displacement was observed in other classical donor-acceptor associations ( $\mathrm{Ni}-\mathrm{Mo}, \mathrm{Co} / \mathrm{W}, \mathrm{Ni} / \mathrm{W}$, etc.) and less classical ones (e.g. $\mathrm{WS}_{2}-\mathrm{Rh}_{2} \mathrm{~S}_{3}$ ) [11]. The reason is that HDS needs more deeply reduced sites, i.e. a larger content of donor $\mathrm{Co}$, $\mathrm{Ni}$, etc. in the catalyst, for increasing in the production of $\mathrm{H}_{\mathrm{so}}$. of such a compound. This has been discussed recently (see especially Refs. [16,20-22]).

The proponents of the CoMoS concept themselves demonstrated that the Mössbauer signal they attributed to the 'CoMoS' species disappeared after a short time (19 h) under the mild reaction conditions of a commercial feed under normal hydrotreating reactions $\left(352{ }^{\circ} \mathrm{C}\right)[23,24]$. Our own results $[25,26]$ and those of others [27], based on different experiments, also demonstrated the same decay of the signal. These results proved the irrelevance of the CoMoS concept, but this does not prove that the hypothesis of a remote control, although reasonable, is the right explanation. In principle, other interpretations are possible.

\subsection{Direct proof of the role of a remote control in hydrotreatment}

The proof was obtained by Gil Llambías et al. in the last years $[20,28,29]$. The test reaction was the HDS activity in the reaction of a commercial gas oil containing $5400 \mathrm{ppm}$ sulfur. The measurements were made under industrial conditions (325-375 ${ }^{\circ} \mathrm{C}, 30 \mathrm{MPa}$ pressure). In the experimental setup, separate beds of materials with different compositions were stacked (Fig. 8). The donor, e.g. $\mathrm{Co}_{9} \mathrm{~S}_{8}$ or other sulfides, was separated from the acceptor $\left(\mathrm{MoS}_{2}\right.$ or $\left.\mathrm{WS}_{2}\right)$ by a substance known to have no activity in the reaction, but which was able to convey spillover hydrogen. We call this layer 'separator' (silica, alumina, silica-aluminas). The results showed the presence of strong synergy [20,28,29]. It is remarkable that the different separators allowed synergetic effects of different magnitudes. These effects can be related to the properties of the hydroxyls present on their surfaces [28]. With silicon carbide that does not possess surface hydroxyls, no synergy was observed because spillover was impossible on the surface. Still more dramatic effects were obtained when the donor and acceptor were impregnated onto a single monolith [28]. In this case, $\mathrm{Co}_{9} \mathrm{~S}_{8}$ and $\mathrm{MoS}_{2}$ were deposited, respectively, at the opposite ends of a monolith while keeping the middle part free from impregnated material. The general conclusion is that a large, probably main, part of the activity increase is due to a remote control exerted by various sulfides of the Group VIII metals.

The remote control mechanism also operates with acid sites. Independent investigations have focused on the action of acid sites on the complex reactions in hydrotreatment. They showed similar effects. Spillover hydrogen created acid sites on a silica-alumina with neither Co nor Mo [30]. The results revealed a parallelism with the role of mobile hydrogen in the case of the zeolites discussed above. The experiments were conducted on mixtures of materials similar to those used in hydrotreatment, i.e., pure silica-alumina 


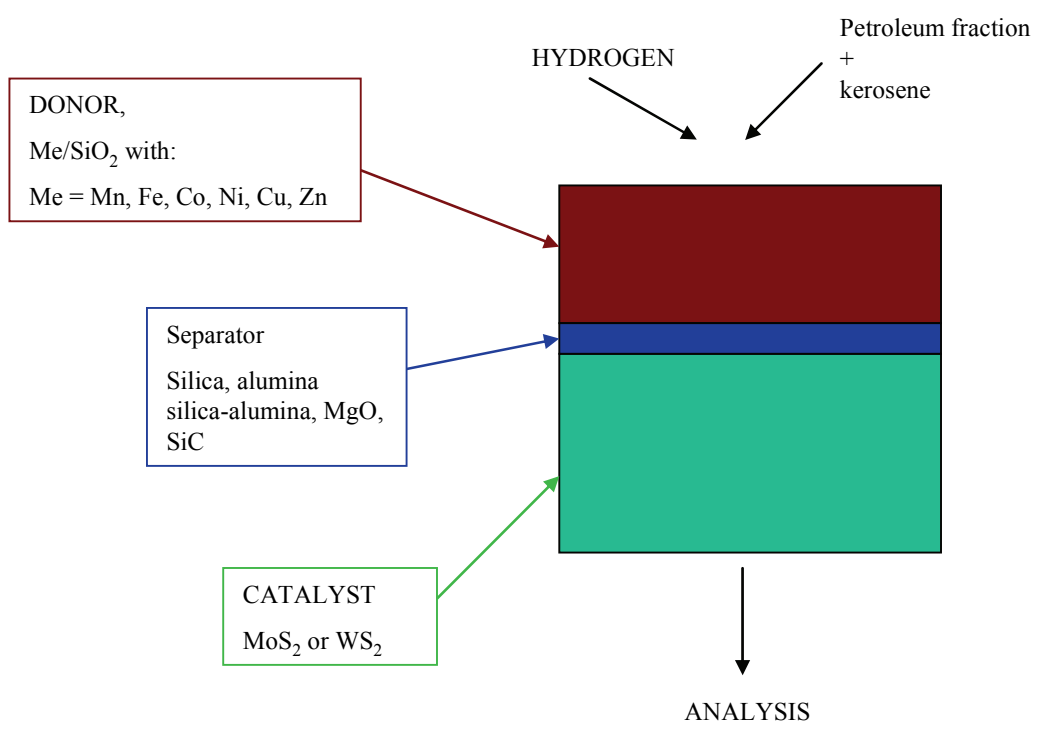

Fig. 8. Remote control in hydrodesulfurization. Principle of the experimental setup for (1) verification of a long distance spillover mechanism, and (2) evaluation of the efficiency of the control as a function of the nature of donors of spillover hydrogen $[28,29]$.

powder and sulfided CoMo supported on a silica powder. Silica has no measurable acidity and no catalytic activity for the reactants. The reactant was diphenylmethane, and the reaction took place under conditions corresponding to those of the industrial process $\left(400{ }^{\circ} \mathrm{C}\right.$ under a pressure of 5 $\mathrm{MPa})$. As in the zeolite case, an increase of the acid function was observed. $\mathrm{H}_{\mathrm{so}}$ was produced by the cobalt sulfide contained in CoMo and it created acid sites. The effect increased with the proportion of CoMo in the mixture. It also increased at higher hydrogen pressure, with the pressure increasing the rate of $\mathrm{H}_{\text {so }}$ production [30]. In this case, as in that of the zeolites in hydrotreatment, Group VIII metals or metal sulfides, especially platinum, exerted this control. With these non-zeolitic catalysts, the $\mathrm{D}-\mathrm{H}$ rate of exchange also increased as well as the intensity of the OD bands in the infrared spectra. This similitude between the effects observed in these hydrotreatment experiments and the case of the zeolites reinforces the credibility of the control of acid sites by spillover hydrogen.

\subsection{Considerations on the mechanism of remote control}

In all the effects already mentioned, the control was exerted by charged species, i.e., hydrogen or oxygen species carrying an electric charge. Before closing this section, it is necessary to add a remark concerning the remote control that will be useful for the next section on the changes of shape. In the case of inorganic catalysts, modifications in the shape are a consequence of the diffusion of these charged species. In the framework of the present contribution concerning the similitude between solid catalysts and enzymes, a natural question is the nature of the signal or messenger in the case of allostery and the corresponding change of shape often observed in enzymes. A hypothesis would be that the messenger is also a small charged species. If this is the case, it would perhaps be possible to discover equivalent remote control mechanisms in the case of enzymes. It is therefore useful to analyze the fundamental causes of the formation of spillover species in solid catalysts. Additional results are mentioned in the next paragraph. They provide a few useful bases for further discussion.

In the field of hydrodesulfurization, Chianelli et al. $[31,32]$ used catalysts prepared in the classical way, i.e., by impregnation of molybdenum together with promoters of the support, followed by sulfidation. They found correlations between the intensity of the synergy and the position in the periodic table of the promoters that increased the activity of the corresponding bimetallic catalysts. They attributed the result to the difference in bond strength of the promoters in their sulfided state. Gil Llambías et al. [20,28,29] have presented a comparison of their results with those of Chanelli et al. for the same elements ( $\mathrm{Mn}, \mathrm{Fe}, \mathrm{Co}, \mathrm{Ni}, \mathrm{Cu}$, and $\mathrm{Zn}$ ). They found that the intensity of the synergism varied in the same way in both works.

Returning to oxidation catalysis, we had noticed that the polarity of the surface of the donor dictated the production rate and/or the value of the partial charge on the spillover species [14]. A ranking of the efficiency of 12 donors was established. In selective oxidation, the number of acceptors to consider is larger than those in hydrotreating, such that, in addition, a ranking of the acceptors is also possible (pp. 203-204 of Ref. [14]). Measurements showed that the 
ionicity/covalency balance of the catalytic oxides dictated their place in the ranking. This highlights the importance of the surface polarity of the donors, which parallels the observations and interpretations proposed in the case of hydrotreatment [29,31,32]. More precisely, the electronic structure of the donor explains the production of $\mathrm{H}_{\mathrm{so}}$ and, consequently, the intensity of the promotion effect.

The same conclusion holds for the cases of selective oxidation or oxidative dehydrogenation as well as for results obtained in two different ways in hydrodesulfurization. Thus, a very surprising observation can be made: the nature of the donor has a comparatively stronger impact on the catalytic activity than the nature of the solid with the active sites (the acceptor). The polarity of the donor is crucial for its effectiveness. By analogy, can we speculate that the control of activity and shape of the enzyme is exerted by charged species from the prosthetic site of enzymes? This will be discussed again at the end of the next section.

\section{Solids that change shape during catalytic reactions as enzymes do}

\subsection{Changes in the shape of an oxidation catalyst when a remote control operates}

The introduction mentioned the fact that the activity of enzymes is often accompanied by modifications in their shape. In his impressive scientific contributions, G. Ertl discovered and interpreted the movements of atoms in two or three near-surface layers of catalysts, i.e., displacements of atoms situated one nanometer or less from the surface.
Actually, many experiments have also shown that structures can be drastically changed at greater depths inside the catalyst structure. Changes of the whole shape of oxidation catalysts have also been mentioned. Our example will consider the partial oxidation of isobutene to methacrolein by molecular oxygen. This reaction is catalyzed by the same $\left\{\mathrm{MoO}_{3} / \mathrm{Sb}_{2} \mathrm{O}_{4}\right\}$ mixture as the reaction of $N$-ethyl formamide discussed above. The same remote control mechanism determines the selectivity of the reaction, as depicted in Fig. 5 , except that oxidation sites are formed instead of $-\mathrm{OH}$ groups. The structure of these sites is more complex than that of the acid sites in the case of $N$-ethyl formamide. At least 4 atoms of molybdenum, 8 atoms of oxygen and at least one $-\mathrm{OH}$ group periodically change position during each of the catalytic cycles. This continuous rocking movement in the structure can propagate to neighboring structural groups. This results in long-range deformations that can be observed with a microscope [33,34]. Initially, the $\mathrm{MoO}_{3}$ crystals in the absence of antimony oxide exhibited smooth faces that remained unchanged after contact with the isobutene-oxygen mixture. Practically no selective oxidation occurred. In an experiment under the same conditions, but with the $\mathrm{MoO}_{3} / \mathrm{Sb}_{2} \mathrm{O}_{4}$ mixture, the remote control mechanism operated, selective oxidation occurred, and steps and groves were progressively formed (Fig. 9) [33]. The crystals at the top of Fig. 9 were several micrometers thick. This showed that the structural changes propagated at distances several thousand times larger than inter-atomic distances. This also showed that spillover triggered the process and continuously activated the structural reorganization at similar distances from the oxygen donor $\mathrm{Sb}_{2} \mathrm{O}_{4}$.

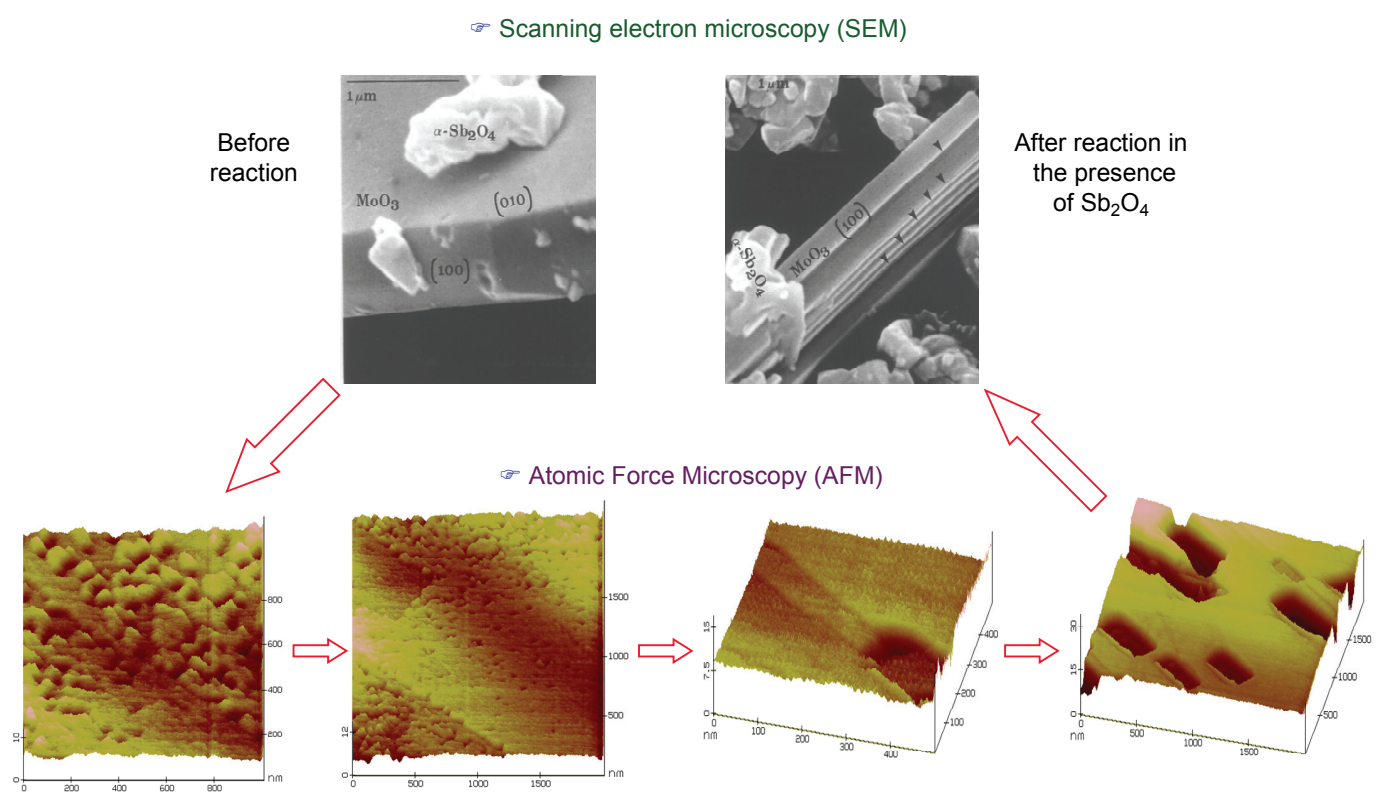

Fig. 9. Electron microscopy investigation of the shape of $\mathrm{MoO}_{3}$ crystallites and their surface: (left) before the catalytic reaction, or after such a reaction in the absence of spillover oxygen, and (right) after the reaction when remote control operates. 
The structure of stoichiometric $\mathrm{MoO}_{3}$ is made up of octahedral $\mathrm{MoO}_{6}$ units connected by the oxygen atoms at their corners (Fig. 10). Sub-oxides of molybdenum $\mathrm{MoO}_{3-x}$ can be formed by the removal of some oxygen atom from the structure without fundamentally modifying the structure. An impressive group of experiments demonstrated that the reaction mechanism consisted of a cycle of reduction of molybdenum oxide by isobutene and re-oxidation of the suboxide by molecular oxygen. This constituted an example of the so-called Mars-Van Krevelen mechanism applicable to most oxidation reactions. The chemical equations (1) and

(2) represent the two steps in the process:

$$
\begin{gathered}
\left(\mathrm{CH}_{3}\right)_{2} \mathrm{CH}=\mathrm{CH}_{2}+\left[\mathrm{MoO}_{3}\right] \rightarrow \mathrm{CH}_{2}=\mathrm{C}\left(\mathrm{CH}_{3}\right) \mathrm{CHO}+\mathrm{H}_{2} \mathrm{O} \\
+\left[\mathrm{MoO}_{3-x}\right] \\
{\left[\mathrm{MoO}_{3-x}\right]+\mathrm{O}_{2} \rightarrow\left[\mathrm{MoO}_{3}\right]}
\end{gathered}
$$

The modification of structure between $\mathrm{MoO}_{3}$ and $\mathrm{MoO}_{3-x}$ is possible because part of the energy is dissipated by the oxidation reaction. This corresponds to the rocking movement described in the previous paragraph. Let us further mention that these changes can be synchronized so that oscillations are sometimes observed in oxidation reactions.

It is possible to analyze in more detail the top pictures of Fig. 9 obtained when $\left[\mathrm{MoO}_{3}\right]$ crystals have been used for some time under the influence of spillover oxygen. Atomic force microscopy makes the phenomenon more spectacular,

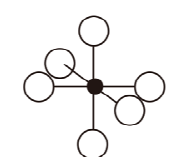

(a)

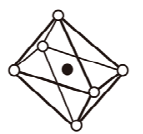

(b)

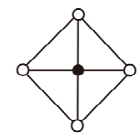

(c)

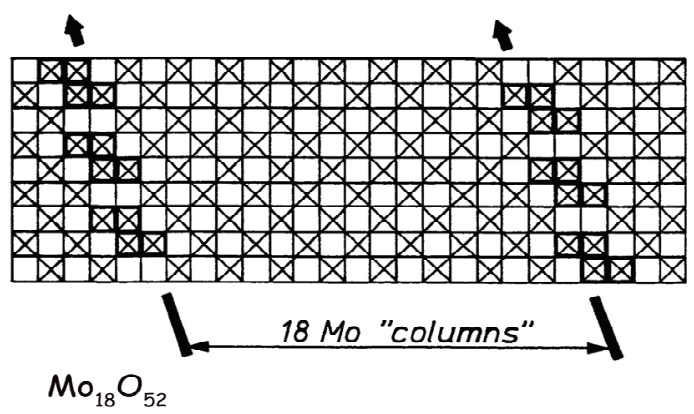

Fig. 10. Structure of $\mathrm{MoO}_{3}$ : the elementary structure is composed of $\mathrm{MoO}_{6}$ octahedra (a), actually distorted octahedra (b), and (c) shown viewed from a corner. This permits the use of a simple picture for describing the overall structure. The bottom of the figure represents the consequence of an abnormal link between octahedra formed when the oxide is sub-stoichiometric in oxygen, e.g., in $\mathrm{Mo}_{18} \mathrm{O}_{52}$. Instead of a connection by the oxygen atoms at the corners, the link is made by the O-O edges common to two adjacent octahedra. Actually, the defects accumulate in a regular way on well defined planes ('shear structures') Unfortunately, the figure cannot show that the octahedra forming each of the four 'defective' building units (in bold black) are then not in the same plane. thanks to its much higher resolution. The bottom of Fig. 9 reveals small pits or holes of nanometer dimensions. The use of crystallites with different aspect ratios (ratios between the faces of different indexes) revealed that the surface of the extended flat planes (010) only had a very low activity, unlike the faces constituting the lateral faces and the walls of the pits. These correspond to (100) planes or planes of similar crystallographic orientations. The selective reaction takes place on these faces, particularly in the pits, that develop a larger surface area. They constitute 'nests' in which the reaction takes place. It is possible to describe the situation by saying that these inorganic catalysts can open their catalytic sites in a way similar to the opening of 'prosthetic sites' in enzymes for receiving the substrates to be transformed. In the case of $\mathrm{MoO}_{3}$ irrigated by spillover oxygen, the active sites may thus be compared to "prosthetic sites'. But they have an additional useful property: their number can be modified. In contrast to prosthetic sites, they continually change their number, shape, and position because of the perpetual rocking of the bonds between octahedra. They remain open all the time.

The changes indicated in equations (1) and (2) are illustrated by Fig. 11. The rocking changes in the links between octahedra, the formation of sub-oxides $\mathrm{MoO}_{3-x}$ and the corresponding long-range defects are indicated.

\subsection{Further mechanistic considerations concerning the alteration of shapes}

With the possible exception of the simplest ones, enzymes continuously change their shape, both when they are 'at rest' and when they are catalyzing reactions. This is due to the Brownian motion of the protein chains that constitute their structure. Shape modification may be so important that the prosthetic site is only accessible, or 'open', to the reactant (the substrate, in the language of enzymology) during a fraction of the time. This is dictated by the statistics of opening and closure. This phenomenon is so important that stabilizing the 'open' configuration is desirable in industry in order to increase turnover. This can be done in different ways.

If the conclusion of section 4.4 holds, the question arises of whether some electric or electronic signal had travelled from the regulating site to the prosthetic site that is usually quite distant. Could the induction effects observed along molecular chains in organic chemistry be sufficient to influence the interactions between protein segments? It may also be asked whether movements of electrically charged species (protons, electrons?) can be involved. Are some polar properties of the controlling site essential for its effectiveness, and thus highlight colloidal properties that exist in addition to the key-in-lock idea that constitutes the classical 


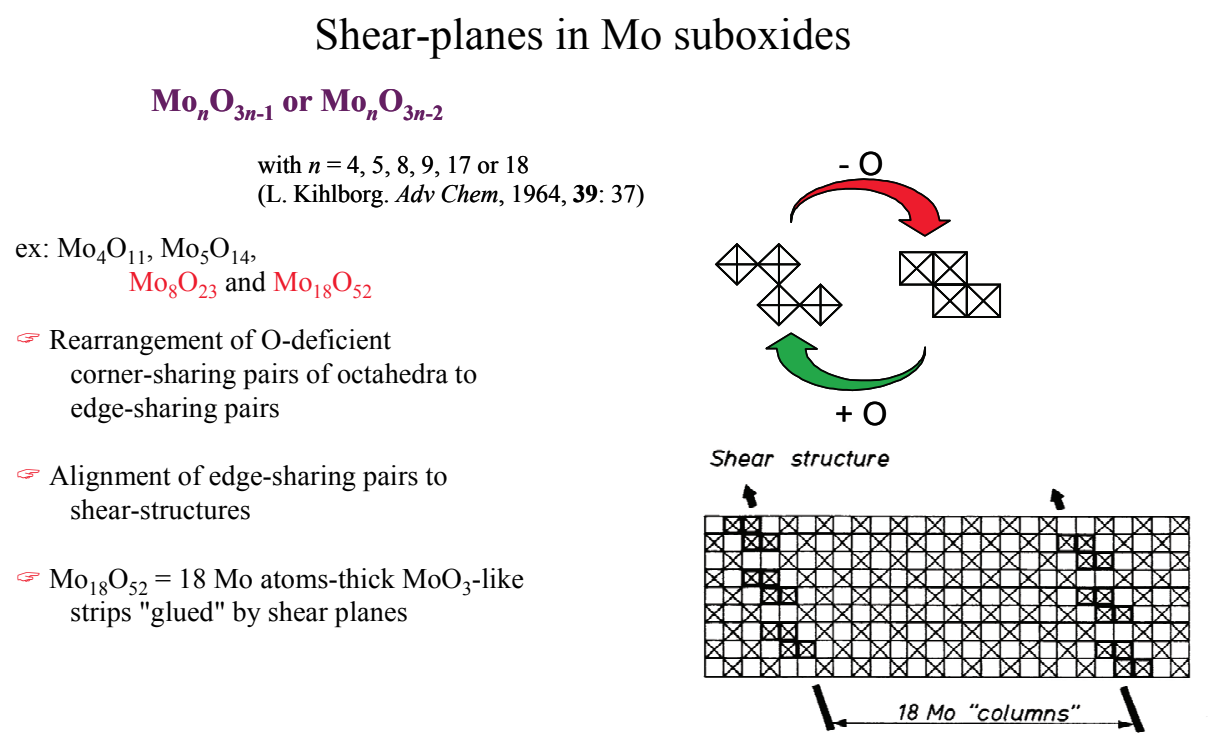

Fig. 11. Summary of the mechanism explaining the sort of plasticity of the surface and bulk of $\mathrm{MoO}_{3}$ observed in the course of a reaction controlled by spillover. The changes are the consequence of the continuous reduction-oxidation cycle of equations (1) and (2). In the continuous rocking changes the link formed in one step of the cycle (e.g. to edge sharing) form a corner sharing link between structural units different from that initially present. The existence of sub-oxides in $\mathrm{MoO}_{3}$ is the key to its catalytic activity. In the cases where they were monitored, structural changes were observed in practically all oxidation and oxidative transformations.

explanation? A conclusion concerning this point would be useful for man-made improvements: more efficiently guiding of the controlling substance towards the prosthetic site despite the continuous modifications in the form of the enzyme. As polarity certainly propagates rapidly along an enzyme protein chain, this can also trigger a complete rearrangement of the whole mutual interaction between these chains.

\section{Prospects: will it be possible to bestow still more 'enzymatic' features onto inorganic catalysts?}

\subsection{General remarks}

Making predictions is the safest way to commit errors. This closing section will therefore mention hopes as well as obstacles. A look at the future constitutes one way, among other ones, to realize new achievements. Scientific considerations certainly constitute incentives.

There is no doubt that successful efforts have already been made to create catalysts that possess the useful properties of solids and adding to them the considerable advantages of enzymes in terms of selectivity. The inconvenience with enzymes and homogeneous catalysts lies in the difficulty of separating them from reaction products. An important line of research is therefore to make hybrids by a combination of solids with organic material such as enzymes or molecules active in homogeneous catalysis. This is currently achieved by grafting the organic partner onto the sur- face of solids. In the case of enzymes, the advantage is the recovery of a very costly catalyst. Actually, the modification is essentially physical. From the scientific point of view, the hope should be more ambitious, which is to change in a fundamental way the chemical catalytic properties. Therefore, the challenge to make solid catalysts more similar to enzymes should consider all changes of properties: resistance to aging, activity, selectivity, etc.

It is possible to distinguish different directions for creating such new catalysts. The approaches are very diverse. However, it is perhaps possible to discern three different lines. These are outlined in the following sections.

\subsection{Design of inorganic structures that imitate those of enzymes}

The zeolites indicated ways to mimick parts of the properties developed by enzymes. A similar trend towards creating enzyme-like catalysts is the possibility to conduct selective reactions for one specific enantiomer in a racemic mixture, and/or selective transformations to asymmetric structures of molecules with no optical activity. Let us recall that one of the simplest example of enantiomers, and certainly the best known, concerns tartaric acid, which exists in two structures, one of which is the mirror image of the other. Understandably, many experiments on enantioselective catalysis used tartaric acid. Presently, two distinct approaches are possible, often using tartaric acid as a model.

A direct approach to finding suitable solids was taken many years ago. It was obvious that inorganic solids whose 
structure has asymmetry, as quartz does, are candidates for a catalyst component. However, the use of quartz as a support for metals active for hydrogenation only led to negligible enantiomeric excesses. Certain zeolitic structures possess cages that are 'asymmetric'. It has not been shown that their use or that of mesoporous inorganic materials has led to any notable success. This is frustrating because if such solids were identified, their production in large quantities is possible by using adequate seeding of the desired optically active form. Less fruitless was the use of solids with 'footprints', or moulds or matrixes, created on the surface of catalytic solids, esp. solid catalysts with acid sites. Footprints can be 'in-printed' when preparing solids, e.g. by precipitation, by including molecules of adequate shapes that can be eliminated afterwards for creating the mould. Several experiments have demonstrated that this approach can be used. Unfortunately, the corresponding catalysts had very poor activity, which was due to many possible reasons. These reasons may be the small number of 'footprints' actually in-printed or that they were not accessible because they were enclosed inside the solid. Another possible cause may be the small probability of encounter with the active site of complicated molecules that have acquired a form adequate for adsorption during the continuous movement of the segments of their molecular chain due to Brownian motion.

\subsection{Protection of enzymatic structures}

One line of research consists of protecting the delicate structure of enzymes and other fragile catalysts by an adequate solid envelope or scaffold. In principle, this can be realized by using the cages of zeolites. For example, some hope has been given to construct phthalocyanine-based catalysts, thus building an artificial oxygen activating material. This would reproduce the properties of certain cytochromes of living organisms. Unfortunately, the sizes of the cavities and phthalocyanine molecules, respectively, were not compatible, with the consequence that either the cages or their content (or both) were damaged or destroyed. New approaches involving only organic synthesis without using pre-existing structures seem to offer promising results, as in the work of P. A. Jacobs and his group in Leuven (Belgium).

\subsection{Developing materials by creating catalytic} cooperation between an inorganic material and (i) an enzyme, or (ii) the active part of an enzyme, or (iii) molecular structures possessing the desired enzymatic property

One important approach involves enantioselective properties. This has been developed by industry. It consists of adding special additives to the reacting medium together with ordinary inorganic catalysts. Tartaric acid has been used, but other molecules are more appropriate. The pharmaceutical and pesticide industries are using this approach. Actually, the way these molecules act is not known with certainty. An approach to solve the problem would be (i) to understand whether the additive gets linked to the molecule that is to be transformed or to the surface of the catalyst before the reaction takes place or (ii) to identify the attachment mechanism, e.g., is it mere molecular recognition or grafting. In both cases, the reaction would be intrinsically enantioselective. This would be a way to go beyond empiricism. But this approach may not lead to materials of the philosophy of this contribution, i.e., obtaining solid catalysts possessing some of the distinctive properties of enzymes without additional components.

Some inorganic solids possess photocatalytic properties and favor specific reactions when properly irradiated by light. The synthesis and study of many new photocatalysts presently constitutes a very active field, in particular for removing harmful molecules from effluents by complete oxidation. Fundamental research suggests that in order to broaden the application field, new materials should be developed that can decompose water and produce hydrogen and oxygen under the action of light. Actually, the photo-dissociation of water is becoming an important goal since the study of the reaction mechanism unveiled the crucial steps following the splitting by light to form intermediate species. Most photocatalysts do not allow a separation of positive and negative species to distances sufficient for hindering extremely rapid recombination. Consequently, photocatalytic solids presently known are not very impressive with respect to water dissociation. However, this separation is made in a very efficient way in plants. The structure of the maze of organic molecules in plant tissue (e.g. leaves) is such that specific 'sinks' exist for each of the charged species. The idea of M. Grätzel in Lausanne (Switzerland) was to combine inorganic photocatalysts with pigments present in plants. This hybrid material was able to separate positive from negative ions and produce sufficient electricity to run a small electric fan. The nature of the bond between the solid photocatalyst and the plant pigment was not known. Other investigators with different aims, in particular to synthesize elaborate organic chemicals, are working along similar lines, e.g. the group of E. Paez in Bucaramanga (Columbia). The electronic interactions between the partners of the hybrid material still need investigations. Some specialists of theoretical chemistry have begun to propose hypotheses but this contribution is not a suitable place for discussing these highly specialized work. The present paragraph and the last remark suggest that creating enzyme-like catalysts based on inorganic solids is a credible development purpose. 


\subsection{The future: other directions not yet actively investigated}

This contribution has presented arguments to make use of the similitude of the catalytic properties of inorganic solids and enzymes. It can be noted that the rigidity of solid constitutes a serious handicap. The elasticity of the cavity walls in zeolites is very small. In spite of their crucial role in increasing activity and selectivity when a remote control mechanism operates, the extent of deformations remains limited. Nevertheless, recent progress in the synthesis of new solids will certainly help overcome this handicap. Using nanotechnology, methods have been developed for producing solids of regular structures that contain inorganic fragments and organic chains. The rational design and production of such solids will allow the elimination of the usual constraints to which purely inorganic structures are subjected. Improvements can occur in several directions. New structures with large pores in a regular array would give controlled access to catalysts in the form of homodispersed inorganic nanoparticles. Chiral organic pillars could shape enantioselective adsorption sites and allow a control of the chirality of adsorbed reactants and/or products. Large molecules often contain lipophilic (hydrophobic) segments together with hydrophilic parts or nanoparticles. A judicious choice of the corresponding sites of the organic pillars can favor or hinder adsorption of the various segments of the reactant and hence modify reaction rates and selectivity. Bifunctional reactions could thus benefit from a pre-positioning of the reactant sites to attack. It is thus logical to predict that organic-inorganic hybrids will have more features of enzymes than present solid catalysts do.

\section{Conclusions}

The conclusion of this article is a mixture of a few pessimistic and many optimistic views concerning the possibility of making inorganic solids that have many of the properties of enzyme, if not all. There is no doubt that an analogy with enzymes will inspire still more efforts towards the creation of better catalysts. The discovery of those that have been outlined in the present work showed that improvements are possible. Possible inspiration triggered by this contribution will certainly need complementary advances in other fields of science and the discovery of new phenomena.

\section{References}

1 Gnep N S, Martin de Amando M L, Guisnet M. In: Pajonk G M, Teichner S J, Germain J E eds. Spillover of Adsorbed Species. Amsterdam: Elsevier, 1983. 309
2 Matsuda T, Fuse T, Kikuchi E. J Catal, 1987, 106: 38

3 Roland U, Winkler H, Steinberg K-H. In: Steinberg K-H Ed. Proceedings of the 2nd International Conference on Spillover. Leipzig: Uni Leipzig, 1989. 63

4 Roland U, Winkler H, Bauch H, Steinberg K-H. J Chem Soc, Faraday Trans, 1991, 87: 3921

5 Roland U, Salzer R, Stolle S. Stud Surf Sci Catal, 1994, 84: 1231

6 Roessner F, Roland U, Braunschweig T. J Chem Soc, Faraday Trans, 1995, 91: 1539

7 Verhoeven W, Delmon B. Bull Soc Chim Fr, 1966: 3065

8 Boldyrev V V, Bulens M, Delmon B. The Control of the Reactivity of Solids. Amsterdam: Elsevier, 1979

9 Oukaci R, Sayari A, Goodwin J H Jr. J Catal, 1987, 106: 318

10 Delmon B. Heterog Chem Rev, 1994, 1: 219

11 Delmon B. Surf Rev Lett, 1995, 2: 25

12 Zhou B, Machej T, Ruiz P, Delmon B. J Catal, 1991, 132: 183

13 Melo-Faus F, Zhou B, Matralis H, Delmon B. J Catal, 1991, 132: 200

14 Weng L T, Delmon B. Appl Catal A, 1992, 81: 141

15 Zabala J M, Mainil M, Grange P, Delmon B. React Kinet Catal Lett, 1975, 3: 285

16 Delmon B. Catal Lett, 1993, 22: 1

17 Delmon B, Froment G F. Catal Rev-Sci Eng, 1996, 38: 69

18 Clausen B S, Mørup S, Topsøe H, Candia R. J Phys Colloq, 1976, 37: 249

19 Topsøe H, Clausen B S, Candia R, Wivel C, Mørup S. J Catal, 1981, 68: 433

20 Baeza P, Ureta-Zanartu M S, Escalona N, Ojeda J , Gil Llambías F J, Delmon B. Appl Catal A, 2004, 274: 303

21 Various contributions. Catal Today, 2008, 130: 1-264

22 Lélias M A, van Gistel J, Maugé F, van Veen J A R. Catal Today, 2008, 130: 109

23 Topsøe H, Candia R, Topsøe N-Y, Clausen B S. Bull Soc Chim Belg, 1984, 93: 783

24 Topsøe H, Clausen B S. Appl Catal, 1986, 25: 273

25 Göbölös S, Wu Q, Delannay F, Grange P, Delmon B, Ladrière J. Polyhedron, 1986, 5: 219

26 Ladrière J, Göbölös S, Delannay F, Delmon B. J Radioanal Nuclear Chem, 1987, 109: 213

27 Breysse M, Fréty R, Vrinat M, Grange P, Genet M. Appl Catal, 1984, 12: 165

28 Baeza P, Villarroel M, Ávila P, López Agudo A, Delmon B, Gil Llambías F J. Appl Catal A, 2006, 304: 109

29 Villarroel M, Baeza P, Escalona N, Ojeda J, Delmon B, Gil Llambías F J. Appl Catal A, 2008, 345: 152

30 Stumbo A M, Grange P, Delmon B. In: Li C, Xin Q eds. Spillover and Migration of Surface Species in Catalysis. Amsterdam: Elsevier, 1997. 211

31 Pecoraro T A, Chianelli R R. J Catal, 1981, 67: 430

32 Harris S, Chianelli R R. J Catal, 1986, 98: 17

33 Gaigneaux E M, Naud J, Ruiz P, Delmon B. In: Grasselli R K, Oyama S T, Gaffney A M, Lyons J E eds. The 3rd World Congress on Oxidation Catalysis. Amsterdam: Elsevier, 1997. 185

34 Gaigneaux E M, Ruiz P, Wolf E E, Delmon B. Appl Surf Sci, 1997, 121: 552 